\title{
Laparoscopic Management of Undescended Testis: Results and Outcomes in a Pediatric Population
}

\author{
C. Kamadjou1,2*, H. E Moby², A. Kameni', E. Muhawenimana3 ${ }^{3}$ F. F. Mouafo Tambo4 ${ }^{4}$ F. Angwafor ${ }^{5}$ \\ ${ }^{1}$ Medical and Surgical Center of Urology, Douala, Cameroon \\ ${ }^{2}$ Faculty of Medicine and Pharmaceutical Sciences, Department of Surgery and Specialties, University of Douala, Douala, Cameroon \\ ${ }^{3}$ Kigali Teaching Hospital, Kigali, Rwanda \\ ${ }^{4}$ Gynaeco Obstetric and Pediatric Hospital, Yaounde, Cameroon \\ ${ }^{5}$ Urology Residency Program, Faculty of Medicine and Biomedical Sciences, University of Yaoundé I, Yaoundé, Cameroon \\ Email: *cyrkamadjou@yahoo.fr
}

How to cite this paper: Kamadjou, C., Moby, H.E., Kameni, A., Muhawenimana, E., Mouafo Tambo, F.F. and Angwafor, F. (2022) Laparoscopic Management of Undescended Testis: Results and Outcomes in a Pediatric Population. Open Journal of Pediatrics, 12, 47-58.

https://doi.org/10.4236/ojped.2022.121006

Received: January 15, 2022

Accepted: February 11, 2022

Published: February 14, 2022

Copyright $\odot 2022$ by author(s) and Scientific Research Publishing Inc. This work is licensed under the Creative Commons Attribution International License (CC BY 4.0).

http://creativecommons.org/licenses/by/4.0/

\section{(c) (i) Open Access}

\begin{abstract}
Aim: This study aimed to evaluate the results of the laparoscopic treatment of high and intra-abdominal undescended testes. Methods: A retrospective study was carried out from 2014 to 2021 at the Centre medico-chirugicale d urologie in Douala. We consulted the clinical records of 27 patients aged 6 15 years who underwent laparoscopic orchiopexy at our hospital. We included patients with unilateral or bilateral undescended testes as detected by palpation and excluded patients with incomplete clinical records. The data collected were entered into Microsoft Excel 2016 and exported to Epi info 7 for analysis. Continuous variables were presented as mean values and standard deviations while categorical variables were presented as frequencies and percentages. The threshold for statistical significance was set at $\mathrm{p}<0.05$. Results: The ages of the 27 participants ranged from 6 years to 15 years, with a mean age of $10.22 \pm 2.68$ years. Hypospadias was an associated abnormality in $7.41 \%$ of participants. The mean duration of the surgical procedure was $80.56 \pm 30.30$ minutes. The overall success rate of the laparoscopic procedure was $95.83 \%$. The mean duration of postoperative hospital stay was $26.67 \pm$ 7.69 hours. The only postoperative complication we encountered was testicular atrophy, which occurred in $7.41 \%$ of participants. All our patients underwent single-stage laparoscopic procedures. Conclusion: Laparoscopy, which is the technique of choice for the diagnosis and management of undescended testis, is more rapid, more effective, and characterized by a shorter hospital stay and fewer postoperative complications compared to open surgery. Single-stage procedures are as effective and safe as two-stage procedures.
\end{abstract}




\section{Keywords}

Undescended Testis, Laparoscopy, Orchiopexy, Orchiectomy, Single-Stage Procedure

\section{Introduction}

The testes are the male genital organs. They are located outside the abdominal cavity in a sac known as the scrotum, where they are maintained at a temperature that is approximately $2.5^{\circ} \mathrm{C}$ less than the normal body temperature [1]. The testes initially develop in the abdominal cavity and then descend into the scrotum. Testicular descent is a complex and multifactorial process [2] that begins by the eighth gestational week [3]. Testicular descent, just like other processes in embryology, could encounter abnormalities. Cryptorchidism, which is defined as the absence of the testes from the scrotal sac [4], is a result of abnormal testicular descent. This abnormality can occur either because the descending testis is arrested in its usual path of descent (true undescended testis) or can migrate from the usual path of descent (ectopic testis) [4]. In this study, we focused on true undescended testis. Undescended testis (UDT) affects $1 \%-6 \%$ of male babies [5]. In most patients, undescended testes migrate into the lower scrotum within the first 3 months of life, probably as a consequence of a postnatal surge of testosterone. Only in less than $1 \%$ of the patients does the testis remain persistently undescended by the age of 1 year. Cryptorchidism occurs four times more commonly unilaterally than bilaterally [5]. Undescended testes are usually classified as low, high, or intra-abdominal, with the intra-abdominal ones being the most common [6]. In approximately $80 \%$ of patients with cryptorchidism, the testis is manually palpable in the inguinal canal [7]. Ultrasonography is the most widely used imaging technique in diagnosing cryptorchidism and locating the undescended testes [8]. However, there are other imaging techniques such as computed tomography, and magnetic resonance imaging [9]. The management of cryptorchidism is usually surgical, via a technique known as orchiopexy [10]. With advancements in technology, this procedure is performed nowadays via laparoscopy. Laparoscopy has the advantages of clearly demonstrating the anatomy and providing visual information upon which a definitive decision can be made for further management; thus, this technique is at the same time diagnostic and therapeutic [11]. In resource-limited settings such as ours, laparoscopic surgery is not a common medical practice, and there is a paucity of data on the efficacy of laparoscopic orchiopexy. Thus, we carried out this study that aimed to evaluate the results of the laparoscopic management of high and intra-abdominal undescended testes.

\section{Patients and Methods}

\section{Patients and data collection}

This was a retrospective study carried out on 27 boys aged 6 - 15 years who un- 
derwent laparoscopic surgery for undescended testes at the Centre medico-chirugicale durologie in Douala, after excluding patients with incomplete clinical records. We consulted the clinical records of these patients and collected data on their ages, testicular findings on physical examination, imaging findings (including ultrasound, computed tomography, and magnetic resonance imaging), associated testicular abnormalities, surgical procedure performed, intra-abdominal pressure during surgery, intraoperative laparoscopy findings, surgical technique used, ultrasound findings at the first and second follow-up appointments (approximately one month and six months after surgery, respectively), testicular location and volume at the first and second follow-up appointments, outcome of the surgical procedures performed, and complications. During the initial physical examinations, we noted the absence of testes from the scrotum and went further to confirm the locations of these absent testes via ultrasound, doppler ultrasound, and even magnetic resonance imaging. We defined testicular atrophy with the help of the testicular atrophy index, which is calculated as follows: TAI $=($ contralateral testis volume-affected testis volume)/contralateral testis volume $\times 100$, with a TAI value of $20 \%$ or more representing testicular atrophy [12].

\section{Surgical procedure and postoperative follow-up}

The aim of this laparoscopic procedure was to restore the testis (orchidopexy) to its normal position in the scrotum or remove the testis completely (orchidectomy) if orchiopexy is not possible. This is to prevent the testis from becoming cancerous, even though the risk of malignancy in such testicular remnants is low [13]. After consulting an anesthesiologist, all patients did laboratory tests, including prothrombin time, kaolin-cephalin time, complete blood count, serum urea and creatinine, and urinalysis. This procedure was performed under general anesthesia. During this procedure, the patient was placed in the dorsal decubitus position and a urinary catheter was placed to facilitate the process of dissection that is much easier when the bladder is empty. Thereafter, pneumoperitoneum was created via insufflation with carbon dioxide at a given pressure that is calculated as follows: Age (in years) $+2 \mathrm{mmHg}$, with a maximum pressure of 14 mmHg. This insufflation could either be done via a mini-laparotomy and insertion of an optical trocar (for younger patients) or by using a Veress needle (for older patients). Thereafter, a one-centimeter arc-shaped incision was made below the umbilicus, followed by either a mini-laparotomy or the use of a Veress needle, depending on the patient's age. Thereafter, the first $5-\mathrm{mm}$ or $10-\mathrm{mm}$ optical trocar was placed, depending on the patient's age. This was followed by a revision of the peritoneal cavity using the $10-\mathrm{mm}$ optical trocar. Two other $5-\mathrm{mm}$ trocars were placed in each iliac fossa, followed by a dissection of the posterior peritoneum and liberation of the spermatic vessels to mobilize the testis. Each vas deferens, spermatic cord, and testicular gubernaculum were identified and isolated while noting their gross anatomy and positions. The gubernaculum testis was identified and mobilized to ensure better clamping and traction of the testes using a pair of forceps. Then, the gubernaculum testis was then sectioned using a pair of monopolar scissors. Dissection was done cranially until a 
sufficient length of the spermatic cord was obtained to perform orchiopexy without tensioning it. The peritoneum was perforated at the lateral face of the round ligament and the anteromedial part of the epigastric vessels. It is worth mentioning that the thirty-degree Trendelenburg position is often necessary to better visualize the testes and their associated structures. If the testis was not found, the procedure was stopped (which was the patient in two of our participants). If the testis was atrophied or vestigial, orchiectomy was performed (this was the patient in three of our study participants). If the testis had a normal appearance, orchiopexy was performed (this was the patient in 22 of our study participants), during which the testis was brought into the lower part of the scrotum and fixed to the scrotal wall. It is worth noting that in all patients, we successfully carried out one-stage laparoscopic procedures (orchiopexy or orchiectomy). We defined the success of the laparoscopic procedure as the presence of the testis in the lower scrotum (normal location) with no atrophy after surgery (for orchiopexy) and the successful removal of the dystrophic testis (for orchiectomy). Highlights of the laparoscopic procedure are presented in Figure 1 and Figure 2.

After the laparoscopic procedure, follow-up appointments were scheduled one month and six months postoperatively. During these follow-up appointments,

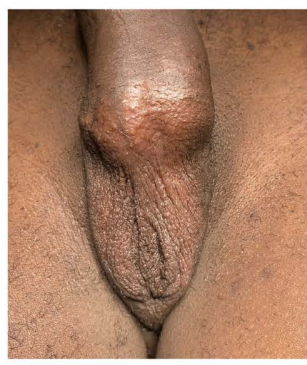

(a)

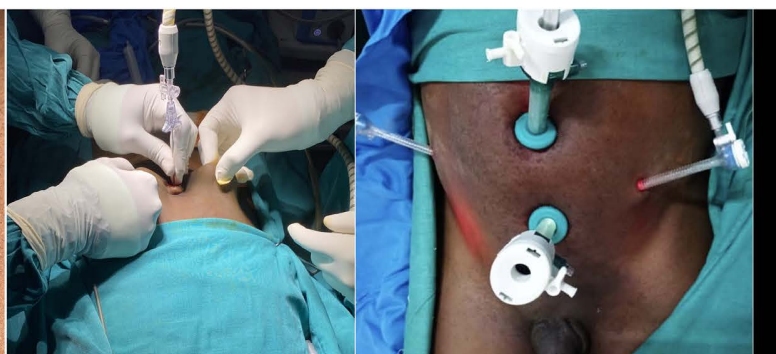

(b) (c)



(d)

Figure 1. (a) Clinical aspect of bilateral undescend testis; (b) Gas insufflation with Veress needle; (c) Position of the trocarts; (d) Intra abdominal view of testis.

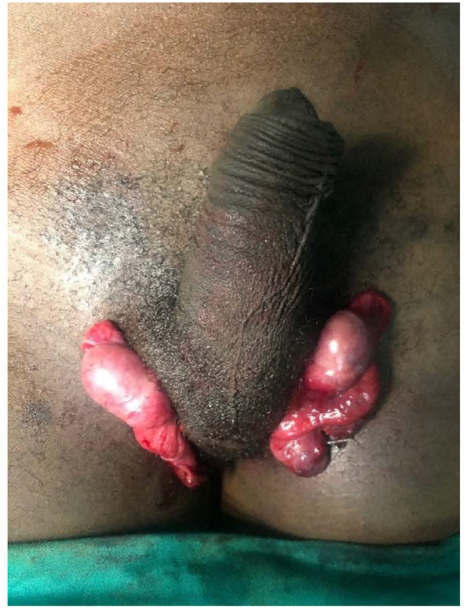

(a)

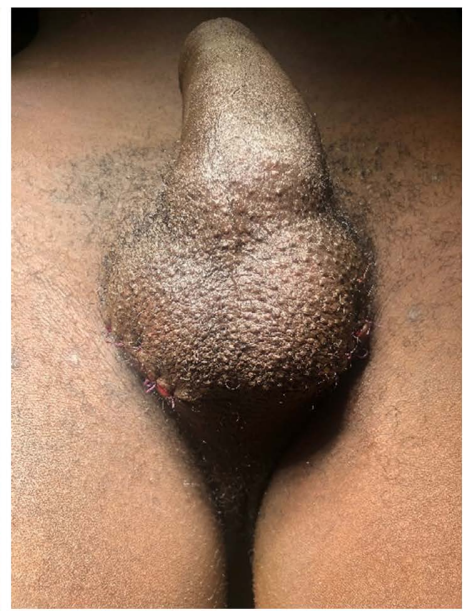

(b)

Figure 2. (a) Intermediate stage of orchiopexy with descended testes visible. (b) Final stage of orchiopexy with both testes in the lower scrotum. 
the patients were examined and ultrasound examination was performed to determine the positions and volumes of the testes.

\section{Data management}

The data collected from patients' clinical records were entered into Microsoft Excel 2016 and exported to Epi info 7 for analysis. Continuous data were presented as mean values and standard deviations for normally distributed data and as median values with interquartile ranges for skewed data. Categorical variables were presented as frequencies and percentages.

\section{Ethical approval}

This study was approved by the institutional review board of the Faculty of Medicine and Pharmaceutical Sciences of the University of Douala and the ethics committee of the Centre medico-chirugicale d urologie in Douala. The requirement for informed consent was waived due to the retrospective nature of the study.

\section{Results}

The ages of the 27 participants ranged from 6 years to 15 years, with a mean age of $10.22 \pm 2.68$ years. The undescended testis was unilateral in 8 (29.63\%) patients and bilateral in 19 (70.37\%) patients. Five of the unilateral cases included the left testis while three involved the right testis. Upon physical examination, the right testis was found in 5 (18.52\%) patients and not found in $22(81.48 \%)$ patients, whereas the left testis was found in $3(11.11 \%)$ patients and not found in $24(88.89 \%)$ patients. Hypospadias was the only identified associated anomaly, and it occurred in $2(7.41 \%)$ of our study participants. Apart from the absence of one or both testes and the presence of hypospadias, there were no other remarkable findings on physical examination in these patients. The patients with hypospadias were later operated upon to correct this congenital anomaly. Imaging modalities (abdominal ultrasound, Doppler ultrasound, and magnetic resonance imaging) were performed. The right testis was found on abdominal ultrasound in 19 (70.37\%) patients, on Doppler ultrasound in $00(0.00 \%)$ patients, on magnetic resonance imaging (MRI) in $2(7.41 \%)$ patients. The left testis was identified on abdominal ultrasound in $22(81.48 \%)$ patients, on Doppler ultrasound in $00(0.00 \%)$ patients, and on MRI in $1(3.7 \%)$ patient. The preoperative characteristics of the study participants are presented in Table 1 . Apart from the locations and sizes (volumes) of the testes, the various imaging techniques revealed no other remarkable findings.

The time-lapse from hospitalization till surgery ranged from 14 days to 67 days, with a mean value of $34.67 \pm 13.20$ days. The intraabdominal pressure during surgery ranged from $8 \mathrm{mmHg}$ to $14 \mathrm{mmHg}$, with a mean value of $11.81 \pm 2.13$ $\mathrm{mmHg}$. Peritoneal insufflation was done via mini-laparotomy in 16 (59.26\%) patients and via the use of a Veress needle in $11(40.74 \%)$ patients. Concerning the findings on laparoscopy, on the right side, a functional testis was found in 19 $(90.48 \%)$ patients and a vestigial testis was found in $2(9.52 \%)$ patients while on 
Table 1. Preoperative characteristics of the study participants.

VARIABLE

Age (years)

$6-10$

$11-15$

Laterality

Unilateral

8 (29.63)

Bilateral

$19(70.37)$

Physical examination findings

Right testis found

Right testis not found

Left testis found

Left testis not found

Associated abnormality

Hypospadias

$2(7.41)$

Imaging methods that identified the right testis

Abdominal ultrasound

Doppler ultrasound

$00(0.00)$

Magnetic resonance imaging

$2(7.41)$

Imaging methods that identified the left testis

Abdominal ultrasound

Doppler ultrasound

$00(0.00)$

Magnetic resonance imaging

the left side, a functional testis was found in $22(95.65 \%)$ patients and a vestigial testis was found in 1 (4.35\%) patient.

As concerns the laparoscopic technique carried out in the patients, right orchiopexy was performed in 19 (90.48\%) patients, right orchiectomy in 2 (9.52\%) patients, left orchiopexy in $22(95.65 \%)$ patients, and left orchiectomy in 1 (4.35\%) patient. The duration of the laparoscopic procedure ranged from 20 minutes to 130 minutes, with a mean duration of $80.56 \pm 30.30$ minutes. The intraoperative details are found in Table 2.

Twenty-four (88.89\%) of the 27 patients were hospitalized for 24 hours after surgery while $3(11.11 \%)$ were hospitalized for 48 hours. The mean postoperative hospitalization duration was $26.67 \pm 7.69$ hours. The time-lapse from surgery to the first follow-up appointment ranged from 25 days to 43 days, with a mean value of $34.04 \pm 4.36$ days. The location of the right testis during the first follow-up appointment was normal (in the lower scrotum) in 23 (95.83\%) patients and abnormal (in the upper scrotum) in $1(4.17 \%)$ patient. For the left testis, the location 
Table 2. Intraoperative details of the study participants.

VARIABLE

FREQUENCY (\%)

Time lapse until surgery (days)

$$
\begin{aligned}
& 0-20 \\
& 20-40 \\
& 40-60
\end{aligned}
$$$$
>60
$$

Intra-abdominal pressure $(\mathrm{mmHg})$

$$
\leq 10
$$

$11-12$

$13-14$

Method of peritoneal insufflation

Mini-laparotomy

Veress needle

Right-side findings

Normal testis

Vestigial testis

Left-side findings

Normal testis

Vestigial testis

\section{Surgical procedure performed}

Right orchiopexy

$19(90.48)$

Right orchiectomy

Left orchiopexy

Left orchiectomy

\section{Duration of surgery (minutes)}

$\leq 60$

$60-120$

$18(66.67)$

$$
>120
$$

was normal in all 25 patients who underwent laparoscopic procedures to the left. During the first follow-up appointment, testicular ultrasound was carried out. The volume of the right testicle on ultrasound ranged from $5 \mathrm{cc}$ to $14 \mathrm{cc}$, with a mean volume of $9.04 \pm 2.65 \mathrm{cc}$. The volume of the left testicle ranged from $6 \mathrm{cc}$ to $14 \mathrm{cc}$, with a mean value of $9.92 \pm 2.36 \mathrm{cc}$.

The time-lapse from surgery to the second follow-up appointment ranged from 177 days to 194 days, with a mean time-lapse of $185.81 \pm 4.57$ days. The mean time-lapse between the two follow-up appointments was $151.78 \pm 4.62$ 
days. The location of the right testis during the second follow-up appointment was normal in $23(95.83 \%)$ patients and abnormal in $1(4.17 \%)$ patient. For the left testis, the location was normal in all 25 patients who underwent laparoscopic procedures to the left. Testicular ultrasound was also performed during the second follow-up appointment. The volume of the right testicle on ultrasound ranged from $2 \mathrm{cc}$ to $13 \mathrm{cc}$, with a mean volume of $9.13 \pm 2.68 \mathrm{cc}$. The volume of the left testicle ranged from $4 \mathrm{cc}$ to $13 \mathrm{cc}$, with a mean value of $9.8 \pm 2.41 \mathrm{cc}$. The volume of one patient's left testis that could not be measured during the first follow-up appointment was measurable during the second follow-up appointment and found to be $12 \mathrm{cc}$. After the second follow-up appointment, the definitive positions and volumes of the testicles were determined. The only postoperative complication we recorded was testicular atrophy, which occurred in two patients (one with left testicular atrophy and the other with right testicular atrophy). The postoperative features of the study participants are presented in Table 3.

\section{Discussion}

This retrospective study aimed to evaluate the results of the laparoscopic treatment of high and intra-abdominal undescended testes. We included 27 patients with a mean age of $10.22 \pm 2.68$ years, which differs from the mean age of 6.7 years reported by Bakr and Kotb [14]. The mean age in our study is higher because of the relative unawareness of this condition in our context. The parents of children with undescended testes often tell us they were reassured by midwives, general practitioners, and even pediatricians that their children's undescended testes will descend with time and that there was no need for any specialized treatment or surgical intervention. Such false information leads to the late diagnosis of the condition. In our study, $70.37 \%$ of patients had bilateral undescended testes, which is similar to the $64.4 \%$ reported by Barbotin et al. in 2019 [15], although it is well known that the prevalence of unilateral undescended testis is approximately four times that of bilateral undescended testis [16]. The misinformation of the population by primary healthcare providers in resource-limited settings like ours is also the main reason for this discrepancy, as unilateral undescended testis is usually considered a benign and self-limiting condition and, as such, children with unilateral undescended testis are often not taken to the hospital. Thus, pediatricians, general practitioners, midwives, and other primary healthcare providers in resource-limited settings should be sensitized about this condition through capacity-building seminars so that cases could be diagnosed and managed early. We identified hypospadias as the only associated abnormality in our patients, occurring in $7.41 \%$ of them. This is in line with the findings of Sabetkish et al. who reported that undescended testis and hypospadias occur concomitantly in $6 \%-31 \%$ of cases [17]. The participants in this study all underwent laparoscopic surgery, which was either orchiopexy or orchiectomy. Orchiectomy was performed in the $4.35 \%-9.52 \%$ of patients who had testicular remnants, which is lower than the $24.2 \%$ reported by Vilijoen et al. in 2020 [18]. The difference in the 
Table 3. Postoperative details of the study participants.

VARIABLE

FREQUENCY (\%)

Duration of hospitalization (hours)

24

$24(88.89)$

48

$3(11.11)$

Right testis location during first follow-up

Normal

$23(95.83)$

Abnormal

Left testis location during first follow-up

Normal

$25(100.00)$

Abnormal

Left testicular volume at first follow-up (cc)

$<10$

$13(54.17)$

$\geq 10$

$11(45.83)$

Right testicular volume at first follow-up

$<10$

$\geq 10$

Right testis location during second follow-up

Normal

$23(95.83)$

Abnormal

Left testis location during second follow-up

Normal

$25(100.00)$

Abnormal

Left testicular volume during second follow-up (cc)

$<10$

$\geq 10$

$11(44.00)$

Right testicular volume during second follow-up

$<10$

$12(50.00)$

$\geq 10$

$12(50.00)$

\section{Complications}

Testicular atrophy

rates of orchiectomy is probably because Vilijoen et al. carried out their study on adolescents and young adults with a mean age of 25.4 years. In this population, the malignant potential of the undescended testes, which increases with age, was higher than that in our study population; as such, the rate of orchiopexy had to be higher since it has been proven that the malignant potential of undescended 
testis increases with age and early orchiectomy significantly reduces the risk of malignancy [19].

The mean duration of the laparoscopic procedure in our study was $80.56 \pm$ 30.30 minutes, which is higher than the $62.50 \pm 15.20$ minutes reported by Yang et al. in 2020 [20]. This difference in surgery duration could be attributed to the fact that Yang et al. are probably more experienced in carrying out this procedure since they handle many more cases than we do, as can be seen by their larger sample size of 256 patients. The overall success rate of the laparoscopic procedure in our study was $95.83 \%$, which is similar to the $96 \%$ reported by Chang et al. in 2001 [21] although they studied both one-stage and two-stage laparoscopic orchiopexy and all our patients underwent one-stage laparoscopic orchiopexy. This is proof of the fact that in the right hands, the one-stage procedure has the same safety and efficacy as the two-stage procedure, as reported by Wang et al. in 2017 [22]. The only postoperative complication we recorded in our study was testicular atrophy, which occurred in $7.4 \%$ of our study participants. This rate is similar to the 5.6\% reported by Jawdat et al. in 2016 [23]. The laparoscopic procedure is also known to be associated with fewer complications, shorter postoperative hospitalizations, and a higher likelihood to be carried out in the outpatient department [24].

\section{Conclusion}

Laparoscopy is the technique of choice for the diagnosis and management of undescended testis. It is more rapid, more effective, and characterized by a short hospital stay and minimal postoperative complications. Laparoscopic surgery should be encouraged in resource-limited settings for better management of undescended testes.

\section{Acknowledgments}

The authors thank Health Search Association for critically reviewing the manuscript.

\section{Authors' Contributions}

\section{Availability of Data and Materials}

The data analyzed in this study are available from the corresponding author upon reasonable request.

\section{Ethics Statement}

Ethical approval was obtained from the institutional review board of the Faculty of Medicine and Pharmaceutical Sciences and the ethics committee of the Centre medico-chirugicale d urologie in Douala, Cameroon. The requirement for informed consent was waived due to the retrospective nature of the study.

\section{Conflicts of Interest}

The authors declare no conflicts of interest regarding the publication of this pa- 
per.

\section{References}

[1] Valeri, A., Mianné, D., Merouze, F., Bujan, L., Altobelli, A. and Masson, J. (1993) Scrotal Temperature in 258 Healthy Men, Randomly Selected from a Population of Men Aged 18 to 23 Years Old. Statistical Analysis, Epidemiologic Observations, and Measurement of the Testicular Diameters. Progrès en Urologie, 3, 444-452.

[2] Husmann, D.A. (2009) Testicular Descent: A Hypothesis and Review of Current Controversies. Pediatric Endocrinology Reviews, 6, 491-495.

[3] Titi-Lartey, O.A. and Khan, Y.S. (2021) Embryology, Testicle. StatPearls Publishing, Treasure Island, FL. http://www.ncbi.nlm.nih.gov/books/NBK557763/

[4] Nepal, P., Kumar, D. and Ojili, V. (2018) Abnormal Descent of the Testis and Its Complications: A Multimodality Imaging Review. South African Journal of Radiology, 22, 1374.

[5] Nah, S.A., Yeo, C.S.W., How, G.Y., Allen, J.C., Lakshmi, N.K., Yap, T.L., et al. (2014) Undescended Testis: 513 Patients' Characteristics, Age at Orchidopexy and Patterns of Referral. Archives of Disease in Childhood, 99, 401-406.

[6] Papparella, A., Parmeggiani, P., Cobellis, G., Mastroianni, L., Stranieri, G., Pappalepore, N., et al. (2005) Laparoscopic Management of Nonpalpable Testes: A Multicenter Study of the Italian Society of Video Surgery in Infancy. Journal of Pediatric Surgery, 40, 696-700.

[7] MacKinnon, A.E. (2005) The Undescended Testis. Indian Journal of Pediatrics, 72, 429-432.

[8] Vijayaraghavan, S.B. (2011) Sonographic Localization of Nonpalpable Testis: Tracking the Cord Technique. Indian Journal of Radiology and Imaging, 21, 134-141.

[9] Tasian, G.E., Copp, H.L. and Baskin, L.S. (2011) Diagnostic Imaging in Cryptorchidism: Utility, Indications, and Effectiveness. Journal of Pediatric Surgery, 46, 2406-2613.

[10] Briefing, P. (2019) Orchiopexy. AORN Journal, 109, 10-12. https://doi.org/10.1002/aorn.12591

[11] Dar, S.A., Bali, R.S., Zahoor, Y., Rashid Kema, A. and Bhardwaj, R. (2018) Undescended Testes and Laparoscopy: Experience from the Developing World. Advances in Urology, 2018, Article ID: 1620470.

[12] Zvizdic, Z., Milisic, E., Halimic, A., Zvizdic, D. and Zubovic, S.V. (2014) Testicular Volume and Testicular Atrophy Index as Predictors of Functionality of Unilaterally Cryptorchid Testis. Medical Archives, 68, 79-82. https://doi.org/10.5455/medarh.2014.68.79-82

[13] Wood, H.M. and Elder, J.S. (2009) Cryptorchidism and Testicular Cancer: Separating Fact from Fiction. The Journal of Urology, 181, 452-461.

[14] Bakr, A.A.M. and Kotb, M. (1998) Laparoscopic Orchidopexy: The Treatment of Choice for the Impalpable Undescended Testis. Journal of the Society of Laparoscopic \& Robotic Surgeons, 2, 259-262.

[15] Barbotin, A.L., Dauvergne, A., Dumont, A., Ramdane, N., Mitchell, V., Rigot, J.M., et al. (2019) Bilateral versus Unilateral Cryptorchidism in Nonobstructive Azoospermia: Testicular Sperm Extraction Outcomes. Asian Journal of Andrology, 21, 445-451. https://doi.org/10.4103/aja.aja 219

[16] Niedzielski, J.K., Oszukowska, E. and Słowikowska-Hilczer, J. (2016) Undescended 
testis-Current Trends and Guidelines: A Review of the Literature. Archives of Medical Science, 12, 667-677.

[17] Sabetkish, S., Kajbafzadeh, A.M. and Sabetkish, N. (2015) Hypospadias and Concomitant Undescended Testis: Comparison of No Skin Incision with Inguinal and Scrotal Skin Incision Orchiopexy. International Journal of Surgery, 22, 154-158.

[18] Viljoen, J.T., Zarrabi, A. and Van der Merwe, A. (2020) Management of Cryptorchidism in Adolescent and Adult Males. African Journal of Urology, 26, Article No. 40. https://doi.org/10.1186/s12301-020-00051-8

[19] Pettersson, A., Richiardi, L., Nordenskjold, A., Kaijser, M. and Akre, O. (2007) Age at Surgery for Undescended Testis and Risk of Testicular Cancer. The New England Journal of Medicine, 356, 1835-1841.

[20] Yang, Z., Li, S., Zeng, H., Yin, J., Xu, W., Li, J., et al. (2020) Laparoscopic Orchiopexy versus Open Orchiopexy for Palpable Undescended Testis in Children: A Prospective Comparison Study. Journal of Laparoendoscopic \& Advanced Surgical Techniques, 30, 453-457.

[21] Chang, B., Palmer, L.S. and Franco, I. (2001) Laparoscopic Orchidopexy: A Review of a Large Clinical Series. BJU International, 87, 490-493.

[22] Wang, C.Y., Wang, Y., Chen, X.H., Wei, X.Y., Chen, F. and Zhong, M. (2017) Efficacy of Single-Stage and Two-Stage Fowler-Stephens Laparoscopic Orchidopexy in the Treatment of Intraabdominal High Testis. Asian Journal of Surgery, 40, 490494.

[23] Jawdat, J.R., Kocherov, S. and Chertin, B. (2016) One-Stage Laparoscopic Orchiopexy for the Treatment of Intraabdominal Testis. The Israel Medical Association Journal (IMA), 18, 669-672.

[24] Suson, K.D., Lakshmanan, Y., Suson, K.D. and Lakshmanan, Y. (2020) Immediate Post-Operative Course Following Open and Laparoscopic Orchiopexy of Abdominal Testes: Is There A Difference? Archive of Urological Research, 4, 17-21. 